\title{
Viewing Classical Radio Galaxies with the Upgraded GMRT and MeerKAT-A Progress Report
}

\author{
Dharam V. Lal ${ }^{1, *} *$, Portia Legodi ${ }^{2}$, Bernie Fanaroff ${ }^{3}$, Tiziana Venturi ${ }^{4} \oplus$, Oleg M. Smirnov ${ }^{2,3}$, Marco Bondi $^{4}(\mathbb{D}$, \\ Kshitij Thorat ${ }^{5,6}$, Landman H. Bester ${ }^{2,3}$ (D) Gyula I. G. Józsa ${ }^{2,3,7}$, Dane Kleiner ${ }^{8}$, Francesca Loi ${ }^{8}$, \\ Sphesihle Makhathini ${ }^{9}$ and Sarah V. White ${ }^{2}$
}

check for

updates

Citation: Lal, D.V.; Legodi, P.; Fanaroff, B.; Venturi, T.; Smirnov, O.M.; Bondi, M.; Thorat, K.; Bester, L.H.; Józsa, G.I.G.; Kleiner, D.; et al. Viewing Classical Radio Galaxies with the Upgraded GMRT and MeerKAT-A Progress Report. Galaxies 2021, 9, 87. https:/ /doi.org/ $10.3390 /$ galaxies 9040087

Academic Editor: Wenwu Tian

Received: 29 September 2021

Accepted: 27 October 2021

Published: 29 October 2021

Publisher's Note: MDPI stays neutral with regard to jurisdictional claims in published maps and institutional affiliations.
1 National Centre for Radio Astrophysics, Tata Institute of Fundamental Research, Post Box 3, Ganeshkhind P.O., Pune 411007, India

2 Department of Physics and Electronics, Rhodes University, P.O. Box 94, Makhanda 6140, South Africa; phetogoportia@gmail.com (P.L.); oms@ska.ac.za (O.M.S.); lbester@ska.ac.za (L.H.B.); gigjozsast@gmail.com (G.I.G.J.); sarahwhite.astro@gmail.com (S.V.W.)

3 South African Radio Astronomy Observatory, 2 Fir Street, Black River Park, Observatory, Cape Town 7925, South Africa; bfanaroff@ska.ac.za

4 INAF-Istituto di Radioastronomia, Via Gobetti 101, I-40129 Bologna, Italy; tventuri@ira.inaf.it (T.V.); bondi@ira.inaf.it (M.B.)

5 Department of Physics, University of Pretoria, Hatfield, Pretoria 0028, South Africa; thorat.k@gmail.com

6 Department of Astronomy, Inter-University Institute for Data Intensive Astronomy, University of Cape Town, Rondebosch, Cape Town 7701, South Africa

7 Argelander-Institut für Astronomie, Auf dem Hügel 71, D-53121 Bonn, Germany

8 INAF-Osservatorio Astronomico di Cagliari, Via della Scienza 5, I-09047 Selargius, Italy; dane.kleiner@inaf.it (D.K.); francesca.loi@inaf.it (F.L.)

9 School of Physics, University of the Witwatersrand, 1 Jan Smuts Avenue, Johannesburg 2000, South Africa; sphemakh@gmail.com

* Correspondence: dharam@ncra.tifr.res.in; Tel.: +91-20-2571-9249

Abstract: We present a progress report of a study of FR I and FR II radio galaxies. Several new morphological features in the radio emission are now revealed using the high $(\mu \mathrm{Jy})$ sensitivity reached in the range 550-1712 MHz, more than a factor of three, at the high $\left(\sim 4^{\prime \prime}-7^{\prime \prime}\right)$ angular resolution with the upgraded Giant Metrewave Radio Telescope (uGMRT) and MeerKAT. Therefore, the aim of this study is to understand if we need to revise our current classification scheme for classical radio galaxies. In order to address our goals, we have carefully constructed a sample of 14 (6 FR I, 6 FR II and 2 FR 0) radio galaxies. The uGMRT and MeerKAT images of our four target sources revealed a wealth of morphological details, e.g., filamentary structure in the emission from the lobes, misalignments, radio emission beyond the hot-spots in three sources, etc.; see Fanaroff et al. (2021). Here, we present preliminary results for two more radio galaxies from our sample using uGMRT, in the light of the local environment. Finally, we are awaiting uGMRT and MeerKAT observations of remaining sample sources. Our results show that for the radio galaxies in this study, the morphological classification scheme for the classical FR I/FR II radio galaxies still holds, even with the improved imaging capabilities of the uGMRT and MeerKAT. Furthermore, we need to be cautious when using automated procedures for classification schemes, e.g., in surveys (with poorer sensitivities and angular resolutions) because of the rich morphological details that are shown in our uGMRT and MeerKAT images.

Keywords: galaxies; active-galaxies; jets-galaxies; nuclei-galaxies; polarisation-galaxies; structureradio continuum; galaxies-galaxies; cluster

\section{Introduction}

It will soon be 50 years since the publication of the paper: The morphology of extragalactic radio sources of high and low luminosity, Fanaroff \& Riley [1]. The paper, with a citation record higher than 2000, has set the basis for the study of extragalactic radio 
sources. The classification of extended extragalactic radio sources in FR I and FR II types is still used to separate low power and high power radio galaxies, respectively [2]. Our knowledge of extragalactic radio sources has improved considerably over the past half century, and the key inferences are summarized below:

(1) Several radio galaxies (e.g., Centaurus A, M 87, NGC 1275, etc.) have been well studied in radio, infrared, optical, X-ray, and $\gamma$-rays (e.g., Centaurus A: [3-5]). They have been detected from radio to $\mathrm{TeV} \gamma$-rays, and also have been studied as high-nutrino and ultra-high energy cosmic-ray potential emitters [6]. These studies propose a leptonic and hadronic model to explain the broadband spectral energy distribution spectrum in them (see also [7] and references therein).

(2) FR I radio galaxies (radio powers, $\mathrm{P}_{\text {rad }}<10^{24} \mathrm{~W} \mathrm{~Hz}^{-1}$ at $1.4 \mathrm{GHz}$ ) with their symmetric prominent jets and lobes, are known to be associated with the low excitation emission line galaxies [8,9]. Departures from the straight FR I radio morphology, are common for optical hosts in galaxy clusters, where the radio jets are exposed to a combination of effects, e.g., galaxy motion through the intracluster medium and "cluster weather" [10], and often form spectacular tailed radio galaxies [11].

(3) FR II radio galaxies $\left(\mathrm{P}_{\text {rad }}>10^{24} \mathrm{~W} \mathrm{~Hz}^{-1}\right.$ at $\left.1.4 \mathrm{GHz}\right)$ are again well known to be associated with large diffuse, double lobes and bright hot spots. The nuclear activity of the optical host usually shows high excitation emission lines [8,9]. The jets of these sources are often asymmetric, and the lobes are the result of backflow from the hot spots.

(4) It is commonly stated that FR I preferentially reside in dense environments, such as clusters and groups of galaxies, whereas FR II are found in less dense environments. Note the famous FR II radio galaxy, Cygnus-A, an exception, is in a galaxy group [12].

(5) FR II radio galaxies populate the Universe to much larger redshifts than FR I radio galaxies [12], which is believed to be partly due to selection effects related to the sensitivity and resolution of the radio interferometers used in surveys. This paradigm is clearly expected to change with the advent of new surveys in the 0.5-2 GHz range, e.g., EMU and superMIGHTEE using ASKAP and uGMRT-MeerKAT, respectively.

(6) The propagation of radio jets of FR I radio galaxies in an external medium is used to explain its morphology and jet broadening $[13,14]$. VLBI studies show that jets of both, FR I and FR II radio galaxies are relativistic (i.e., [15,16]). However, jets in FR I decelerate closer to the core than jets in FR II, possibly due to differences in their environments.

(7) Last but not least, a new class of low power radio galaxies has been recently characterized, the so-called FR 0 [17]. The radio power of these sources is typical of FR I radio galaxies; however, they are compact on the scale of few arcseconds. High sensitivity observations at high angular resolution show that some of them have double-sided jets on very small angular scales, but many remain compact.

In the recent times, the amazing performances of the radio interferometers, which are paving the way to the Square Kilometre Array (e.g., see [18-20]), are posing new exciting questions, which challenge our knowledge in the field. Some of them are as follows:

(1) Does the "simple" FR I/FR II classification of morphology still hold up in the era of arrays with very high dynamic range?

(2) Does very low surface brightness diffuse radio emission beyond the known boundaries of radio galaxies exist that is associated with the radio galaxy itself?

(3) Is it possible that the dominant FR I/FR II classification holds for the current cycle of activity, but low surface brightness emission has been blown away by winds or buoyancy into more complex (and so far missed) shapes?

(4) What is the nature of FR 0 , and how do they fit into the overall classification? 


\section{Sample Selection}

In order to address the questions highlighted in Section 1, we selected a sample of FR I and FR II radio galaxies from the $4 \mathrm{C}$ catalogue $\left(-7^{\circ}<\delta<80^{\circ}\right.$; [21]) that meet the following selection criteria. The source

(i) Is hosted by a detected optical galaxy;

(ii) Has spectroscopically determined redshift $z$, and $0.05<z<0.20$; thereby to (i) have a similar fraction of FR Is and FR IIs and to (ii) ensure detection of Mpc scale extended emission using uGMRT 550-850 MHz band (band-4);

(iii) Is in the declination range $\left[-10^{\circ}:+20^{\circ}\right]$, to ensure comparable $(u, v)$-coverages and visibility with both, uGMRT and MeerKAT arrays; and

(iv) Has a clear double radio morphology at the $\left(45^{\prime \prime}\right)$ angular resolution of the NRAO VLA Sky Survey (NVSS: [22]).

These well defined selection criteria provided us with a total of 14 sources; 6 FR I, 6 FR II and 2 FR 0 radio galaxies.

\section{Observations and Data Reduction}

The upgraded Giant Metrewave Radio Telescope (uGMRT), an array with a hybrid configuration $[23,24]$, was used to observe our six samples during cycle-36, and the remaining eight sample sources will be proposed for uGMRT observations in the forthcoming GTAC cycles. Similarly, MeerKAT is also a hybrid array [25], which was used to observe ten sample sources during A01 and A02 cycles, and the remaining four sources will be proposed during the next MeerKAT call for proposal. The observations described in this paper were all carried out with the new dual polarization (RR and LL) 550-850 MHz band (band-4) receivers of $\mathrm{uGMRT}$, and with the dual linear polarization (horizontal and vertical) 856-1712 MHz (L-band) receivers [26,27] of MeerKAT.

The data reduction and calibration of the data presented in this paper was carried out following the standard procedures [28,29]. Our choice of sample sources, along with on-source integration time per source for the uGMRT and the MeerKAT arrays, were such that we obtained nearly identical $(u, v)$-coverages, and hence imaging sensitivities. Thus, the MeerKAT and uGMRT data cover a frequency range from 550 to $1712 \mathrm{MHz}$ providing radio images at nearly identical angular resolutions and sensitivities, which is ideal for studying the radio morphology and spectral substructures in the jets and radio lobes of the carefully selected sample of radio galaxies.

\section{Results}

Since this is an ongoing project, we here present our understandings of radio galaxies in three parts, the (recent) past (see also [28]), the present and the future, corresponding to the status of uGMRT and MeerKAT observations of the well defined sample of 14 radio galaxies.

\subsection{The Past}

Fanaroff et al. [28] presented the results for the first set of four radio galaxies from this study and concluded that at least for the study of four sources, the classical FR I/FR II morphological classification still holds with the current improved imaging capabilities, but the richness in details also suggests caution in the systematic morphological classification carried out with automatic procedures in surveys with poorer sensitivity and angular resolution. While the authors concluded that the overall FR I-FR II classification scheme still holds, at least for our targets, the combination of $\mu \mathrm{Jy} \mathrm{beam}^{-1}$ sensitivity and high $\left(\sim 5^{\prime \prime}\right.$ to $\left.7^{\prime \prime}\right)$ angular resolution over the full $550-1712 \mathrm{MHz}$ range reveals very interesting features. Below, we provide inferences from this early study.

\subsubsection{Radio Morphology}

Figures 1 and 2 provide the high-sensitivity, high-resolution images of four sources using uGMRT and MeerKAT, respectively. Clearly, it demonstrates that in almost all 
cases, barring CGCG 044-046, the surface brightness declines sharply at the edges of the visible radio lobes and of the low surface brightness features. Below, we present salient morphological features of images of our four radio galaxies shown in Figures 1 and 2.
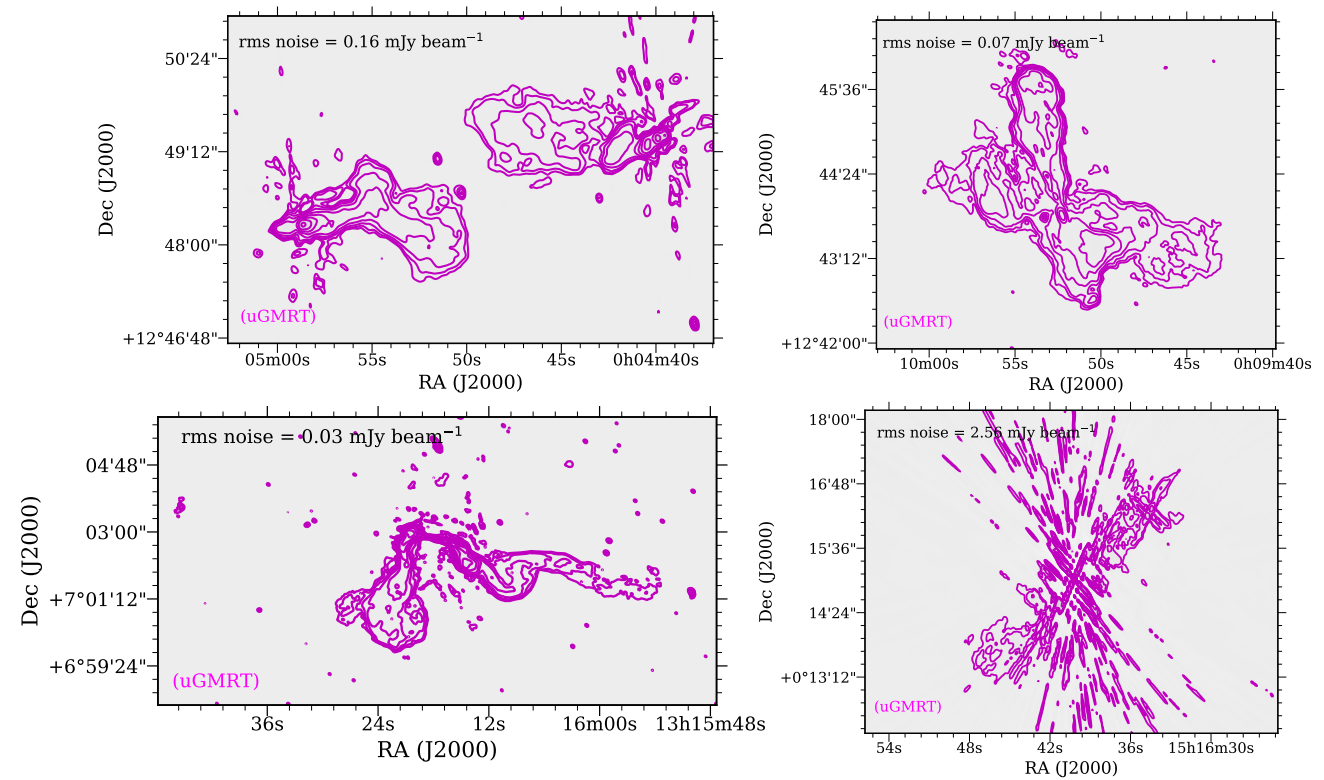

Figure 1. The uGMRT images of our four sample sources, 4C 12.02 (top-left panel), 4C 12.03 (top-right panel), CGCG 044-046 (bottom-left panel) and CGCG 021-063 (bottom-right panel). The images have angular resolutions of $\sim 4^{\prime \prime}$. The magenta surface brightness radio contours, correspond to the full synthesis uGMRT images, and the levels are RMS $\times 3,6,12, \ldots, 384$, which increases by a factor of two. The RMS noise levels in the near vicinity of the source are provided on the upper-left corner of each image. Note that the image of CGCG 021-063 is dynamic range limited, most likely because of the strong compact core.

4 C 12.02-It is a FR II radio galaxy whose optical host galaxy is located at redshift $z=0.143$. The angular resolution of the uGMRT provides insight into the morphology of the hot spots, while the MeerKAT highlight the details and extent of the radio lobes (see also [28] and Figures 1 and 2, top-left panel images). The hot spots are resolved to show multiple peaks. More specifically, there is a minimum of two hot spots near the termination of the west jet, and the hot spot before the bow shock is extended in an arc perpendicular to the source major axis, which may suggest some sort of reflected, internal shock.

4C 12.03 - It is an X-shaped radio source that is associated with an elliptical host galaxy, classified as a low-emission line radio galaxy located at redshift $z=0.156[30,31]$. Our new uGMRT and MeerKAT images suggest that northern and southern jets leading to the north and south hot spots, respectively, form the active axis, whereas the east-west axis forms the low-surface brightness wings (see also [30] and Figures 1 and 2, top-right panel images). The north-south radio morphology seems to follow an arc, i.e., it shows asymmetry. In addition, the angular resolution of our uGMRT and MeerKAT images clearly shows the presence of two inner brightness peaks, forming an inner double lobed structure, that are perfectly aligned with the outer large north-south radio lobes. Thus, the source is possibly a restarted radio source [32].

CGCG 044-046- The radio galaxy is identified as a cD galaxy and is associated with the Zwicky cluster $1313.7+0721$ at $z=0.050145$. We discuss this source (see Figures 1 and 2, bottom-left panel images), including its spectral and polarization properties in Section 4.1.2 below.

CGCG 021-063-It is a triple extended or a S-/Z-shaped radio source that is located at redshift $z=0.0525$. Our uGMRT and MeerKAT images are dynamic range limited because of the dominant compact radio core (see Figures 1 and 2, bottom-right panel images). However, images show that the radio emission has two components, a radio galaxy that 
is embedded in a low-surface brightness cocoon of radio emission. The radio lobes have characteristic FR II morphology, and only one (visible) jet that is pointing towards us.
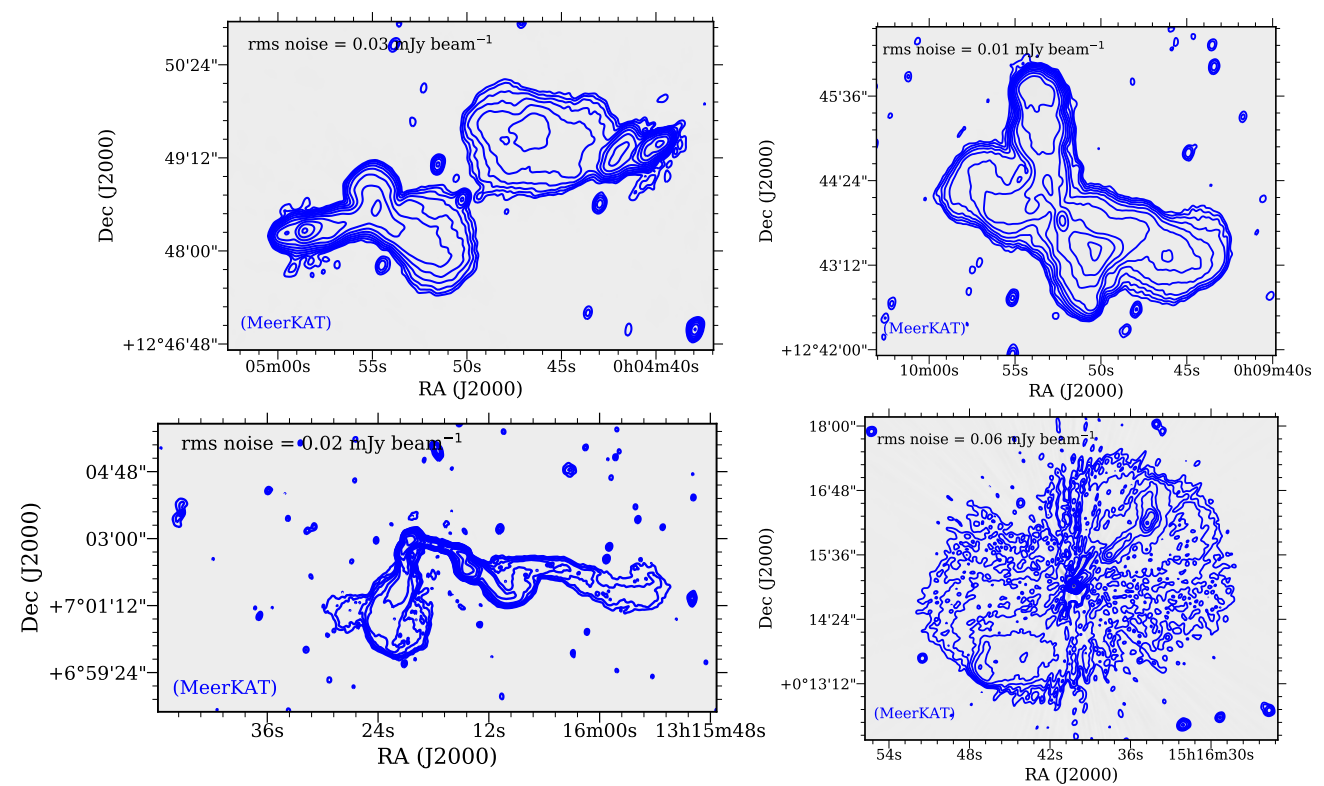

Figure 2. The MeerKAT images of our four sample sources, 4C 12.02 (top-left panel), 4C 12.03 (topright panel), CGCG 044-046 (bottom-left panel) and CGCG 021-063 (bottom-right panel). The images have angular resolutions of $\sim 7^{\prime \prime}$. The blue surface brightness radio contours, correspond to the full synthesis MeerKAT images, and the levels are RMS $\times 3,6,12, \ldots, 384$, which increases by a factor of two. The RMS noise levels in the near vicinity of the source are provided on the upper-left corner of each image. Note that the uGMRT images (see Figure 1) and MeerKAT images are remarkably similar in detail, which gives us confidence in image fidelity, imaging processes and calibration of the uGMRT and MeerKAT data.

\subsubsection{CGCG 044-046}

A bent radio source, typical of radio galaxies at the centre of galaxy clusters and groups $[11,33]$. The uGMRT and MeerKAT images suggest that it possibly belongs to the wide-angle tail class of radio galaxies (see Figures 1 and 2, bottom-left panel images). The extension of the western tail was also detected earlier at low-radio frequencies via GLEAM, though at a much lower angular resolution [34-36]. Images show an inner pair of straight jets, similar to the radio jets of FR I radio galaxies, which form two hot spots. Our images also show another bend at the southern end of the tail, behind the radio tail. Furthermore, there is a sharp transition between the western jet and the tail, i.e., the tail shows very little transverse expansion, makes a few wiggles and then fades completely (see [28]).

In case of observed properties of CGCG 044-046 source, we believe that the local environment plays a major role, e.g., the physical mechanism giving rise to bent tails is possibly due to the motion of the host galaxy through the ICM. The total intensity and spectral index images using uGMRT and MeerKAT highlight three different regions of emission beyond the compact flat spectrum core, namely, (1) the inner jets reaching out all the way to the hot spots; (2) the central region of the radio tails and (3) the terminating part of the radio tails (see also [28]).

Spectral properties - The eastern lobe seems to bend behind the radio emission, which is called the eastern protrusion (see [28] labelled 'A' in Figure 5, left-left panel image), has a steep spectral index $=-1.6 /-1.8$ located at the far end of the radio tail. Whereas in the western emission, the transition from the central to the terminating part of the tail (region 3 ) is sharp both in the total intensity image and in the spectral index image, which steepens from moderate values $\approx-1.0$ to must steeper values $\approx-1.5$. In the eastern lobe, we note a flatter central ridge with $\alpha \approx-0.7$ surrounded by steeper emission with $\alpha \approx-0.9$ and 
a sharp spectral transition with similar values is seen beyond the eastern hot spot. The inner jets and the hot spots (region 1 ) have a fairly uniform flat spectral index, $\simeq-0.6$. The transition between the inner jet and the central part of the tail is also sharp in the western part of the radio galaxy, both in the total intensity image and in the spectral index image, which steepens to $\alpha \simeq-0.9$. This part of the tail (region 2 ) shows prominent bending with edge brightening. We are unclear of the origin of these sharp transitions in surface brightnesses and spectral properties, though these could be due to intermittent activity in the radio emission of the AGN and/or significant interaction of the jets and lobes with discontinuities in the IGM.

Polarisation properties-We were fortunate to have included the polarization calibration source in our MeerKAT observation for this source. The figure presented in (Fanaroff et al. [28] Figure 8, lower panel image), shows the electric field vectors superimposed on the MeerKAT total intensity image. We note remarkable asymmetry in polarization between the western and eastern regions of the radio emission and the two tails differ significantly in polarization fraction. Patnaik, Malkan \& Salter [37] using VLA Land $C$-band constrained the rotation measures across the source to within $\pm 30 \mathrm{rad} \mathrm{m}^{-2}$. Along similar lines, the central and terminating part of the western tail is much more polarized than the south-eastern one in our images and our recovered polarization vectors are consistent with Patnaik, Malkan \& Salter [37]. Furthermore, this radio galaxy is located close to the center of a galaxy cluster, with dense ICM in its vicinity, we therefore believe that CGCG 044-047 is not in the plane of the sky and the Laing-Garrington effect [38] is responsible for the observed polarization asymmetry [28].

\subsection{The Present}

The uGMRT and MeerKAT images presented in Fanaroff et al. [28] cover a frequency range from $550 \mathrm{MHz}$ to $1712 \mathrm{MHz}$ at nearly identical angular resolutions and sensitivities. This early study demonstrated that these observing bands of the uGMRT and MeerKAT together form an ideal situation for studying the radio morphology and spectral structures in the jets and radio lobes of radio galaxies. Here, we present the observations of two sample radio sources, $4 \mathrm{C}-03.43$ and 3C 105 using the uGMRT at 550-850 MHz (band-4) and present salient features of their radio morphologies.

$4 C-03.43$ - The radio source located at $z=0.05196$ hosted by an elliptical host galaxy [39]. Our uGMRT image (see Figure 3, left panel image) shows the radio morphology of a classic FR I radio galaxy. It seems that the radio jets connecting the outer lobes are curved in a way that suggests a rotation of the radio galaxy rather than motion through the external medium.

Another radio source of an unusual morphology associated with the WISEA J113325.24-040414.6 source is located $\sim 6$ arcmin south-east of our target $4 \mathrm{C}-03.43$ radio galaxy. It is detected in the NVSS (flux density $=10.6 \pm 1.3 \mathrm{mJy}$ ) and is also detected at the low-frequency GLEAM survey (flux density = $254 \pm 46 \mathrm{mJy} ;[22,34]$ ).

$3 C 105-A$ radio source located at $z=0.10308$ hosted by an highly flattened elliptical red galaxy, and is a typical NLRG with only nuclear emission lines [40]. The historical radio source, first classified as a type 2 Seyfert galaxy and subsequently as a highly absorbed hyperluminous FR II radio source. Our uGMRT image (see Figure 3, right panel image) is dynamic range limited due to the dominant southern hot spot; nevertheless, the radio morphology consists of a weak core and prominent hot spots. A careful inspection suggests a ridge of emission having an overall curved shape within the southern radio lobe, and the northern radio lobe has a characteristic 'hammer-head' structure. This characteristic feature could be interpreted in terms of the recent escape of the lobe from a sharply bounded halo around the host galaxy [41]. 

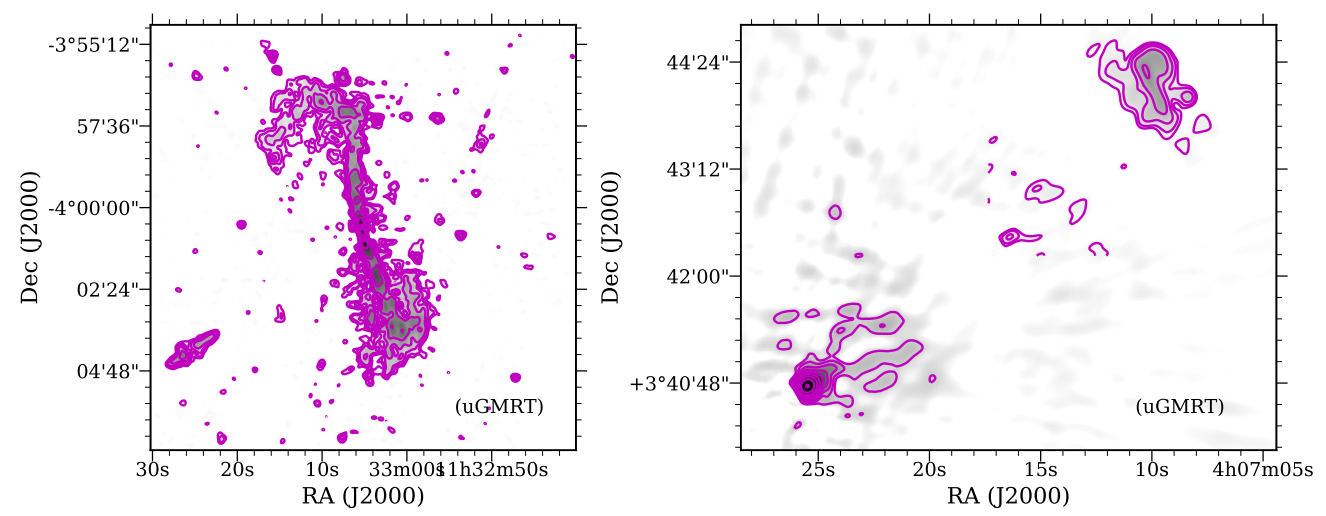

Figure 3. The images of our two sample sources, 4C -03.43 (left panel) and 3C 105 (right panel) at 550-850 MHz of the uGMRT. The uGMRT images have native angular resolutions of $\sim 4^{\prime \prime}$, which are convoluted using $8^{\prime \prime}$ Gaussian kernel. The surface brightness contour levels, in magenta, are RMS $\times$ $3,6,12, \ldots$, which increases by a factor of two. The rms noise values are $33.8 \mu \mathrm{Jy}_{\text {beam }}{ }^{-1}(4 \mathrm{C}-03.43$, left panel image) and $126.1 \mu \mathrm{Jy}$ beam $^{-1}$ (3C 105 right panel image) at the half-power points in the two images, respectively. Note that the lowest two radio contours surrounding the southern radio lobe of 3C 105 (right panel image) have been suppressed to highlight morphological features of the northern radio lobe showing characteristic 'hammer-head' structure (see also Section 4.2 for a discussion), and to show a confirm detection of the radio core; otherwise, we see the starry pattern around dominant southern hot spot. Such a pattern was also seen in our CGCG 021-063 radio galaxy (see Figure 1, bottom-right panel image) to some extent; thus, the image is dynamic range limited and we are exploring improved imaging algorithms to address this.

\subsection{The Future}

It is clear that at present, we are limited by the statistics. Hence, the observations of the complete sample of 14 (6 FR I, 6 FR II and 2 FR 0 ) radio galaxies that are carefully selected with well defined selection criteria is a must in order to address our key goal: if the differences between the FR I and FR II morphologies still hold with the improved imaging capabilities of the current generation of radio interferometers, or if we need more morphological classes. The observations of the remaining sample sources are proposed for early 2022, and the project is therefore still under discussion, so stay tuned.

\section{Conclusions}

In this paper, we have summarized the results presented in Fanaroff et al. [28] for the uGMRT and MeerKAT images of four radio galaxies belonging to a larger sample of 14 (6 FR I, 6 FR II and 2 FR 0) radio galaxies that are carefully selected with well defined selection criteria that cover a frequency range from $550 \mathrm{MHz}$ to $1712 \mathrm{MHz}$, more than a factor of three, providing radio images at nearly identical angular resolutions and sensitivities. We have also presented new uGMRT images of two more radio galaxies from our sample at $550-850 \mathrm{MHz}$ band and we are awaiting data for the remaining sample sources in order to address our scientific aims. These images for the first time also reveal several new sources in the fields of view in addition to deep images of our targets sources. Our work further shows that very good image sensitivity over a broad range of angular scales is necessary to perform a detailed study of radio galaxies.

The uGMRT and MeerKAT images of our first four radio galaxies are remarkably similar in detail, which not only gives us confidence in image fidelity, imaging processes and calibration of the uGMRT and MeerKAT data, but also suggests that the overall FR I-FR II classification scheme still holds. In addition (i) our data reveal very interesting morphological features for radio galaxies at $\mu \mathrm{Jy}$ beam ${ }^{-1}$ sensitivity and high $\left(\sim 5^{\prime \prime}\right.$ to $\left.7^{\prime \prime}\right)$ angular resolution over the full 550-1712 MHz range; (ii) the $856-1712 \mathrm{MHz}$ band spectral structure and polarization information using MeerKAT for CGCG 044-046 suggests that the source is not in the plane of the sky and that the observed properties are strongly 
affected by the cluster environment and we interpret the asymmetries in the polarization properties as due to the Laing-Garrington effect. Finally, our preliminary results for two radio galaxies (i) suggest a rotation of the radio galaxy rather than motion through the external medium in case of $4 \mathrm{C}-03.43$ and (ii) show limitation of uGMRT data, e.g., in 3C 105 radio galaxy, which needs attention using improved imaging algorithms.

Clearly, for surveys made with radio telescopes that have much lower sensitivity to diffuse, low brightness emission, we warn against the accuracy of the morphological classification of radio galaxies made with source detection algorithms. Therefore, in order to perform a detailed study of radio galaxies, a good image sensitivity over a broad range of angular scales for a statistically large sample is necessary. Our observations using uGMRT and MeerKAT over a broad frequency coverage for the remaining sample sources would statistically attempt to address this and several more outstanding and challenging questions.

Author Contributions: Project conceptualisation, B.F., D.V.L., T.V. and O.M.S.; methodology, B.F., D.V.L., T.V. and O.M.S.; data reduction, D.V.L. and O.M.S.; data analysis and investigations, B.L., D.V.L., T.V., O.M.S., M.B. and K.T.; caracal pipeline developers, O.M.S., K.T., P.L., L.H.B., G.I.G.J., D.K., F.L., S.M. and S.V.W.; writing-original draft preparation, B.F., D.V.L., T.V. and O.M.S.; writingreview and editing, B.F., D.V.L., P.L., T.V., O.M.S., M.B., K.T., L.H.B., G.I.G.J., D.K., F.L., S.M. and S.V.W.; project administration, B.F. All authors have read and agreed to the published version of the manuscript.

Funding: DVL acknowledges the support of the Department of Atomic Energy, Government of India, under project no. 12-R\&D-TFR-5.02-0700. TV acknowledges the support from the Ministero degli Affari Esteri e della Cooperazione Internazionale, Direzione Generale per la Promozione del Sistema Paese, Progetto di Grande Rilevanza ZA18GR02. OS's research is supported by the South African Research Chairs Initiative of the Department of Science and Technology and National Research Foundation. DK acknowledges funding from the European Research Council (ERC) under the European Union's Horizon 2020 research and innovation programme (grant agreement no. 679627). FL acknowledges financial support from the Italian Minister for Research and Education (MIUR), project FARE, project code R16PR59747, project name FORNAX-B. SVW acknowledges the financial assistance of the South African Radio Astronomy Observatory.

Institutional Review Board Statement: Not applicable.

Informed Consent Statement: Not applicable.

Data Availability Statement: The GMRT data underlying this article are available via the GMRT online archive facility ${ }^{1}$ The MeerKAT data will be publicly available via the SARAO archive ${ }^{2}$ (Proposal ID SCI-20190418-BF-01) after the end of the proprietary period in mid-2021, but may be shared earlier upon reasonable request to the corresponding author. All data analyses packages used in this work are publicly available, and their URLs have been noted in the main text.

Acknowledgments: We thank our referees for useful comments that helped to improve the paper. We thank the staff of the GMRT who made these observations possible. The GMRT is run by the National Centre for Radio Astrophysics of the Tata Institute of Fundamental Research. The MeerKAT telescope is operated by the South African Radio Astronomy Observatory, which is a facility of the National Research Foundation, an agency of the Department of Science and Innovation. We are grateful to the full MeerKAT team at SARAO for their work on building and commissioning MeerKAT.

Conflicts of Interest: The authors declare no conflict of interest.

\section{Notes}

https://naps.ncra.tifr.res.in/goa/data/search[naps.ncra.tifr.res.in], 26 October 2021.

2 https://archive.sarao.ac.za[archive.sarao.ac.za], 26 October 2021. 


\section{References}

1. Fanaroff, B.L.; Riley, J.M. The morphology of extragalactic radio sources of high and low luminosity. Mon. Not. R. Astron. Soc. 1974, 167, 31P-36P. [CrossRef]

2. Blandford, R.; Meier, D.; Readhead, A. Relativistic Jets from Active Galactic Nuclei. Annu. Rev. Astron. Astrophys. 2019, 57, 467-509. [CrossRef]

3. Fraija, N.; Aguilar-Ruiz, E.; Galván-Gámez, A.; Marinelli, A.; de Diego, J. Study of the PeV neutrino, $\gamma$-rays, and UHECRs around the lobes of Centaurus A. Mon. Not. R. Astron. Soc. 2018, 481, 4461-4471. [CrossRef]

4. Hardcastle, M.J.; Worrall, D.M.; Kraft, R.P.; Forman, W.R.; Jones, C.; Murray, S.S. Radio and X-Ray observations of the jet in Centaurus A. Astrophys. J. 2003, 593, 169-183. [CrossRef]

5. Hardcastle, M.J.; Croston, J.H. Radio galaxies and feedback from AGN jets. NewAR 2020, 88, 1539-1581. [CrossRef]

6. Fraija, N.; Marinelli, A. Neutrino, $\gamma$-ray, and cosmic-ray fluxes from the core of the closest radio galaxies. Astrophys. J. 2016, 830, 81-93. [CrossRef]

7. IceCube Collaboration. Evidence for high-energy extraterrestrial neutrinos at the IceCube detector. Science 2013, $342,1-38$.

8. Best, P.N.; Heckman, T.M. On the fundamental dichotomy in the local radio-AGN population: Accretion, evolution and host galaxy properties. Mon. Not. R. Astron. Soc. 2012, 421, 1569-1582. [CrossRef]

9. Heckman, T.M.; Best, P.N. The Coevolution of Galaxies and Supermassive Black Holes: Insights from Surveys of the Contemporary Universe. Annu. Rev. Astron. Astrophys. 2014, 52, 589-660. [CrossRef]

10. Burns, J.O.; Hanisch, R.J.; White, R.A.; Nelson, E.R.; Morrisette, K.A.; Moody, J.W. A VLA 20 cm Survey of Poor Groups of Galaxies. Astron. J. 1987, 94, 587-809. [CrossRef]

11. Lal, D.V.; Rao, A.P. 3C 129: The GMRT observations. Astron. Astrophys. 2004, 420, 491-489. [CrossRef]

12. Hardcastle, M.J. Jets, hotspots and lobes: What X-ray observations tell us about extragalactic radio sources. Phil. Trans. R. Soc. 2005, 363, 2711-2727. [CrossRef]

13. Bicknell, G.; Jones, D.L.; Lister, M. Relativistic jets. NewAR 2004, 48, 1151-1155. [CrossRef]

14. Laing, R.A.; Bridle, A.H. Relativistic jet models for two low-luminosity radio galaxies: Evidence for backflow? Mon. Not. R. Astron. Soc. 2012, 424, 1149-1169. [CrossRef]

15. Giovannini, G.; Cotton, W.D.; Feretti, L.; Lara, L.; Venturi, T. VLBI Observations of a Complete Sample of Radio Galaxies: 10 Years Later. Astrophys. J. 2001, 552, 508-526. [CrossRef]

16. Venturi, T.; Castaldini, C.; Cotton, W.D.; Feretti, L.; Giovannini, G.; Lara, L.; Marcaide, J.M.; Wehrle, A.E. VLBI Observations of a Complete Sample of Radio Galaxies. VI. The Two FR I Radio Galaxies B2 0836+29 and 3C 465. Astrophys. J. 1995, 454, 735-762. [CrossRef]

17. Baldi, R.D.; Capetti, A.; Giovannini, G. High-resolution VLA observations of FR0 radio galaxies: The properties and nature of compact radio sources. Mon. Not. R. Astron. Soc. 2019, 482, 2294-2304. [CrossRef]

18. Hardcastle, M.J.; Croston, J.H.; Shimwell, T.W.; Tasse, C.; Gürkan, G.; Morganti, R.; Murgia, M.; Röttgering, H.J.A.; van Weeren, R.J.; Williams, W.L. NGC 326: X-shaped no more. Mon. Not. R. Astron. Soc. 2019, 488, 3416-3422. [CrossRef]

19. Ramatsoku, M.; Murgia, M.; Vacca, V.; Serra, P.; Makhathini, S.; Govoni, F.; Smirnov, O.; Andati, L.A.L.; De Blok, E.; Józsa, G.I.G.; et al. Collimated synchrotron threads linking the radio lobes of ESO 137-006. Astron. Astrophys. 2020, 636, L1-L5. [CrossRef]

20. Schellenberger, G.; Vrtilek, J.M.; David, L.; O'Sullivan, E.; Giacintucci, S.; Johnston-Hollitt, M.; Duchesne, S.W.; Raychaudhury, S. NGC 741-Mergers and AGN Feedback on a Galaxy-group Scale. Astrophys. J. 2017, 845, 84-97. [CrossRef]

21. Pilkington, J.D.H.; Scott, P.F. A survey of radio sources between declinations $20^{\circ}$ and $40^{\circ}$. Mem. R. Astron. Soc. 1965, 69, 183-224.

22. Condon, J.J.; Cotton, W.D.; Greisen, E.W.; Yin, Q.F.; Perley, R.A.; Taylor, G.B.; Broderick, J.J. The NRAO VLA Sky Survey. Astron. J. 1998, 115, 1693-1716. [CrossRef]

23. Gupta, Y.; Ajithkumar, B.; Kale, H.S.; Nayak, S.; Sabhapathy, S.; Sureshkumar, S.; Swami, R.V.; Chengalur, J.N.; Ghosh, S.K.; Ishwara-Chandra, C.H.; et al. The upgraded GMRT: Opening new windows on the radio Universe. Curr. Sci. 2017, 113, 707-714. [CrossRef]

24. Swarup, G.; Ananthakrishnan, S.; Kapahi, V.K.; Rao, A.P.; Subrahmanya, C.R.; Kulkarni, V.K. The Giant Metre-Wave Radio Telescope. Curr. Sci. 1991, 60, 95.

25. Jonas, J.; MeerKAT Team. The MeerKAT Radio Telescope. MeerKAT Sci. Pathw. SKA 2016, PoS, 1-22.

26. Lehmensiek, R.; Theron, I.P. On reflector feeds with unidirectional axially symmetric radiation patterns: Their cross-polarization performance and efficiencies. In Proceedings of the 31st URSI General Assembly and Scientific Symposium (GASS), Beijing, China, 16-23 August 2014; pp. 1-4.

27. Lehmensiek, R.; Theron, I.P. Deriving an Optimum Mapping Function for the SKA-Shaped Offset Gregorian Reflectors. In Proceedings of the International Conference Electromagnetics in Advanced Applications (ICEAA), Cape Town, South Africa, 2-7 September 2012; pp. 321-324.

28. Fanaroff, B.; Lal, D.V.; Venturi, T.; Smirnov, O.M.; Bondi, M.; Thorat, K.; Bester, L.H.; Józsa, G.I.G.; Kleiner, D.; Loi, F.; et al. A new look at old friends - I. Imaging classical radio galaxies with uGMRT and MeerKAT. Mon. Not. R. Astron. Soc. 2021, 505, 6003-6016. [CrossRef]

29. Lal, D.V. Upgraded GMRT Observations of the Coma Cluster of Galaxies: The Observations. Astrophys. J. Suppl. Ser. 2020, 250, 22-31. [CrossRef] 
30. Lal, D.V.; Rao, A.P. Giant Metrewave Radio Telescope observations of X-shaped radio sources. Mon. Not. R. Astron. Soc. 2007, 374, 1085-1102. [CrossRef]

31. Cotton, W.D.; Thorat, K.; Condon, J.J.; Frank, B.S.; Józsa, G.I.G.; White, S.V.; Deane, R.; Oozeer, N.; Atemkeng, M.T.; Bester, L.; et al. Hydrodynamical backflow in X-shaped radio galaxy PKS 2014-55. Mon. Not. R. Astron. Soc. 2020, 495, 1271-1283. [CrossRef]

32. Schoenmakers, A.P.; Mack, K.H.; De Bruyn, A.G.; Röttgering, H.J.A.; Klein, U.; Van Der Laan, H. A new sample of giant radio galaxies from the WENSS survey. II. A multi-frequency radio study of a complete sample: Properties of the radio lobes and their environment. Astron. Astrophys. 2000, 146, 293-322. [CrossRef]

33. Ramatsoku, M.; Verheijen, M.A.W.; Kraan-Korteweg, R.C.; Jarrett, T.H.; Said, K.; Schröder, A.C. A near-infrared study of the obscured 3C129 galaxy cluster. Astron. Astrophys. 2020, 644, 107-139. [CrossRef]

34. Hurley-Walker, N.; Callingham, J.R.; Hancock, P.J.; Franzen, T.M.O.; Hindson, L.; Kapińska, A.D.; Morgan, J.; Offringa, A.R.; Wayth, R.B.; Wu, C.; et al. GaLactic and Extragalactic All-sky Murchison Widefield Array (GLEAM) survey - I. A low-frequency extragalactic catalogue. Mon. Not. R. Astron. Soc. 2017, 464, 1146-1167. [CrossRef]

35. White, S.V.; O Franzen, T.M.; Riseley, C.J.; Wong, O.I.; Kapińska, A.D.; Hurley-Walker, N.; Callingham, J.R.; Thorat, K.; Wu, C.; Hancock, P.; et al. The GLEAM 4-Jy (G4Jy) Sample: I. Definition and the catalogue. Publ. Astron. Soc. Aust. 2020, 37, 18-74.

36. White, S.V.; Franzen, T.; Riseley, C.J.; Wong, O.I.; Kapińska, A.D.; Hurley-Walker, N.; Callingham, J.R.; Thorat, K.; Wu, C.; Hancock, P.; et al. The GLEAM 4-Jy (G4Jy) Sample: II. Host galaxy identification for individual sources. Publ. Astron. Soc. Aust. 2020, 37, 17-73. [CrossRef]

37. Patnaik, A.R.; Malkan, M.A.; Salter, C.J. Multifrequency observations of the wide-angle tail radio source 1313+073. Mon. Not. R. Astron. Soc. 1986, 220, 351-362. [CrossRef]

38. Garrington, S.T.; Leahy, J.P.; Conway, R.G.; Laing, R.A. A systematic asymmetry in the polarization properties of double radio sources with one jet. Nature 1988, 331, 147-149. [CrossRef]

39. Rector, T.A.; Stocke, J.T.; Ellingson, E. Quasar Radio Morphology and Clustering Environment at $z \sim 1 / 2$. Astron. Astrophys. 1995, 110, 1492-1497. [CrossRef]

40. Madrid, J.P.; Chiaberge, M.; Floyd, D.; Sparks, W.B.; Macchetto, D.; Miley, G.K.; Axon, D.; Capetti, A.; O’Dea, C.P.; Baum, S.; et al. Hubble Space Telescope Near-Infrared Snapshot Survey of 3CR Radio Source Counterparts at Low Redshift. Astrophys. J. Suppl. Ser. 2006, 164, 307-333. [CrossRef]

41. Leahy, J.P.; Black, A.R.S.; Dennett-Thorpe, J.; Hardcastle, M.J.; Komissarov, S.; Perley, R.A.; Riley, J.M.; Scheuer, P.A.G. A study of FRII radio galaxies with $z<0.15-$ II. High-resolution maps of 11 sources at $3.6 \mathrm{~cm}$. Mon. Not. R. Astron. Soc. 1997, $291,20-53$. 